\title{
Sympathetic ophthalmia in fellow eye after vitrectomy for massive subretinal hemorrhage secondary to polypoidal choroidal vasculopathy
}

This article was published in the following Dove Press journal: International Medical Case Reports Journal

\section{Tetsuyuki Suetsugu'}

Tsutomu Yasukawa ${ }^{2}$

Akiyoshi Uemura ${ }^{1,2}$

Masahiko Higaki'

Yuichiro Ogura ${ }^{2}$

'Department of Ophthalmology, Jyuzen Kinen Hospital, Hamamatsu, Shizuoka, Japan; ${ }^{2}$ Department of Ophthalmology and Visual Science, Nagoya City University Graduate School of Medical Sciences, Nagoya, Aichi, Japan
Correspondence: Tsutomu Yasukawa Department of Ophthalmology and Visual Science, Nagoya City University Graduate School of Medical Sciences, I Kawasumi, Mizuho-cho, Mizuho-ku, Nagoya, Aichi 467-860I, Japan

$\mathrm{Tel}+8|52853825|$

Fax +81528419490

Email yasukawa@med.nagoya-cu.ac.jp

\begin{abstract}
We experienced a case of sympathetic ophthalmia in a fellow eye after vitrectomy for subretinal hemorrhage related to polypoidal choroidal vasculopathy. A 60-year-old male consulted us for polypoidal choroidal vasculopathy with subretinal hemorrhage in his left eye. The recurrence of massive subretinal hemorrhage refractory to repeated pars plana vitrectomies leads to phthisis bulbi. Two months later, multiple serous retinal detachments were observed in his right eye. Positive human leukocyte antigen-DR4 and the uveitis were helpful in distinguishing between sympathetic ophthalmia and age-related macular degeneration. High-dose pulse intravenous steroid contributed to recovery of visual acuity after resolution of serous retinal detachment.
\end{abstract}

Keywords: sympathetic ophthalmia, polypoidal choroidal vasculopathy, vitrectomy, vitreous hemorrhage, uveitis

\section{Introduction}

Sympathetic ophthalmia is a rare, bilateral granulomatous uveitis that occurs after a penetrating trauma or a surgery. ${ }^{1-3}$ The time from trauma to onset of uveitis varies between patients, ranging from a few days to a few decades, although $90 \%$ of cases occur within a year after a triggering injury to an eye. ${ }^{1}$ Sympathetic ophthalmia shares some similar clinical, genetic, and histopathological features with Vogt-Koyanagi-Harada (VKH) disease since both are autoimmune disorders targeting the melanocytes. ${ }^{4-7}$ Currently, a retinal surgery is an emerging risk factor for sympathetic ophthalmia. ${ }^{8-10}$ We describe a case where the onset of sympathetic ophthalmia is possibly associated with the vitrectomy performed to treat subretinal hemorrhage from polypoidal choroidal vasculopathy (PCV) in an exciting eye.

\section{Case report}

A 60-year-old Japanese male presented for upper visual field defect in the left eye in June 2015. His best-corrected visual acuity (BCVA) was 20/17 in the right eye and $20 / 22$ in the left eye. There were no signs of chronic inflammation in both eyes, such as mutton-fat keratic precipitates, anterior chamber flare, and posterior synechiae, at initial presentation. In the left eye, massive submacular hemorrhage, sub-retinal pigment epithelial (RPE) hemorrhage, and an orange-red lesion characteristic to PCV were observed (Figure 1A, B). Fluorescein angiographic image obtained using Optos $^{\circledR}$ California (Optos, Dunfermline, Scotland, UK) manifested hyperfluorescent area corresponding to the orange-red lesion and surrounding blocked fluorescence 
due to sub-RPE hemorrhage (Figure 1C). Also, indocyanine green angiographic image obtained using Heidelberg Retina Angiography 2 (HRA ${ }^{\circledR}$; Heidelberg Engineering GmbH, Heidelberg, Germany) showed focal hyperfluorescence in the area of polypoidal lesion (Figure 1D). Because of complicated vitreous hemorrhage, 25 -guage pars plana vitrectomy combined with phacoemulsification and intraocular lens implantation was performed in July 2015. In November 2016, bullous hemorrhagic RPE detachment and marginal subretinal hemorrhage recurred (Figure 1E), accompanied by vitreous hemorrhage. The patient underwent second 25-guage vitrectomy with silicone oil tamponade in December 2016. High intraocular pressure and hyphema persisted postoperatively and the left eye led to phthisis bulbi despite the third and fourth vitrectomies. No tissue plasminogen activator, gas, or cryotherapy was used during any of the surgeries.

In February 2017, the patient presented with a complaint of blurred vision in his right eye. He had tinnitus and headache with hypersensitivity of scalp from 2 weeks before the onset of blurred vision. The BCVA was 20/17 in the right eye and no light perception in the left eye. In the right eye, slit-lamp biomicroscopy showed cells in the anterior chamber. Fundus examination revealed multiple serous retinal detachments with a subretinal septum (Figure 2A, B). Fluorescein angiography revealed a broad area of hyperfluorescence in the posterior pole and optic disc during the late phase (Figure 2C). Human leukocyte antigen (HLA)-DR4 was positive, and the patient was diagnosed as sympathetic ophthalmia.

Since the BCVA of the right eye dropped to 20/67 due to serous retinal detachments, a pulse steroid therapy (intravenous methylprednisolone $1,000 \mathrm{mg} /$ day for 3 days) followed by oral prednisolone $40 \mathrm{mg} /$ day was initiated. Two weeks later, the BCVA improved to 20/20. In May 2017, disappearance of serous retinal detachments was confirmed by optical coherence tomography (Figure 2D). Oral prednisolone was tapered gradually until December 2017 and was discontinued. No recurrence was observed thereafter. A written informed consent was obtained from the patient for use of his medical record data and images in this case report. The institutional approval was not required for publication of the case report.

\section{Discussion}

We observed a case of sympathetic ophthalmia, which may be associated with vitrectomy performed to treat subretinal hemorrhage due to PCV. According to a population-based study conducted in the United Kingdom, the estimated risk of sympathetic ophthalmia after vitrectomy was 1 in 799 eyes $(0.125 \%)$, which was higher than 1 in 1,357 eyes $(0.074 \%)$ after external retinal detachment surgery. ${ }^{9}$ Another study from

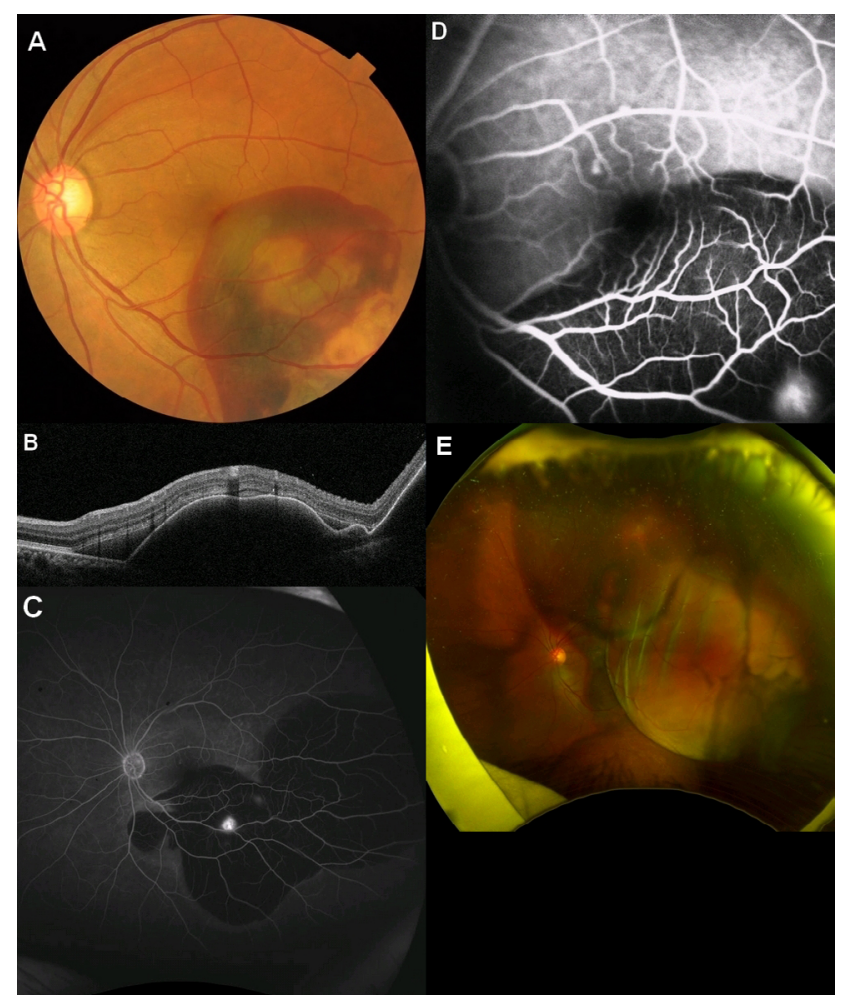

Figure I Massive subretinal hemorrhage and vitreous hemorrhage due to PCV in the left eye.

Notes: (A) Fundus photograph of the left eye at first visit showing subretinal hemorrhage in the posterior pole extending to the lower arcade and an orange-red lesion. (B) Optical coherence tomography of the left eye showing subretinal hemorrhage and sub-RPE hemorrhage due to PCV. (C) Optos ${ }^{\circledR}$ image of fluorescein angiography showing hyperfluorescent area corresponding to the orange-red lesion of PCV and surrounding blocked fluorescence due to sub-RPE hemorrhage. (D) HRA $2^{\circledR}$ image of indocyanine green angiography showing focal hyperfluorescence in the area of polypoidal lesion. (E) Optos image of the left eye 16 months after the first vitrectomy showing bullous hemorrhagic RPE detachment and marginal subretinal hemorrhage. Massive vitreous hemorrhage occurred about a week after the image was obtained.

Abbreviations: PCV, polypoidal choroidal vasculopathy; RPE, retinal pigment epithelial.

the United Kingdom and Republic of Ireland reported that the minimum incidence of sympathetic ophthalmia was 0.03 per 1,00,000 people. Of those reported, pars plana vitrectomy was the sole cause of sympathetic ophthalmia in $33 \%$ of the patients. ${ }^{10}$ The risk of patients developing sympathetic ophthalmia may rise due to increase in number of vitrectomies performed globally in recent years.

We carefully ruled out the possibility of the bilateral occurrence of age-related macular degeneration and other inflammatory diseases. Electrocardiogram and chest X-ray were normal. Laboratory blood test showed white blood cell count $8,560 / \mu \mathrm{L}, \mathrm{C}$-reactive protein $0.01 \mathrm{mg} / \mathrm{dL}$, angiotensin I converting enzyme $13.8 \mathrm{IU} / \mathrm{L}$, lysozyme $3.9 \mathrm{IU} / \mathrm{L}$, Treponema pallidum hemagglutination negative, fluorescent treponemal antibody absorption negative, T-SPOT ${ }^{\circledR}$.TB (Oxford Immunotec Ltd., Abingdon, Oxfordshire, UK) negative, 


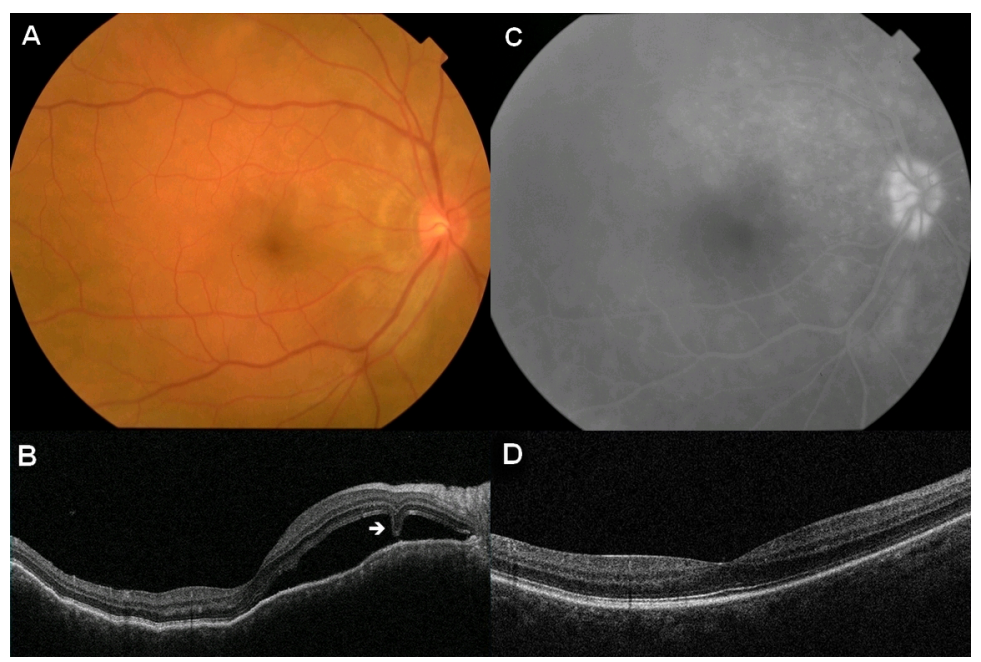

Figure 2 Serous retinal detachments due to sympathetic ophthalmia in the right eye.

Notes: (A) Fundus photograph of the right eye showing serous retinal detachments in the posterior pole. (B) OCT of the right eye showing serous retinal detachment. An arrow indicates subretinal septum, which is the characteristic of sympathetic ophthalmia. (C) Late-phase fluorescein angiography shows a broad area of hyperfluorescence in the posterior pole and optic disc. (D) OCT of the right eye after a cycle of pulse steroid therapy and oral prednisolone administration showing disappearance of serous retinal detachment.

Abbreviation: OCT, optical coherence tomography.

Bartonella henselae deoxyribonucleic acid negative, and HLA-DR4 positive. B. henselae was examined to rule out cat scratch disease from a pet cat that the patient had. The test results were consistent with the previous reports that Japanese patients with sympathetic ophthalmia and VKH disease were prone to express HLA-DR4, HLA-DRw53, and HLA-Bw54. ${ }^{1,6,7}$ Both diseases share similar clinical, genetic, and histopathological features, such as Dalen-Fuchs nodule (inflammatory choroidal nodules manifested as white dots in the fundus), similar HLA expression patterns, and T-cellmediated autoimmune response..$^{1,3-7}$ HLA-DR4 expression in our case was one of the supporting evidences for making a diagnosis of sympathetic ophthalmia.

A pulse steroid therapy followed by high-dose oral prednisolone was an effective treatment option for sympathetic ophthalmia. Currently, systemic steroid administration is considered to be the primary therapy. Oral prednisone $1.0-2.0 \mathrm{mg} / \mathrm{kg} /$ day tapered gradually over $3-4$ months is often administered as an initial treatment. Also, a cycle of pulse steroid therapy, ie, intravenous administration of methylprednisolone $1,000 \mathrm{mg} /$ day for 3 days, is often administered in severe cases. ${ }^{1}$ In our case, the patient was treated with a cycle of pulse steroid therapy followed by oral prednisolone administration. We consider that systemic steroid treatments have successfully contributed to preventing bilateral blindness from prolonged inflammation in our patient.

Currently, an enucleation is the best prevention against sympathetic ophthalmia. ${ }^{1}$ In a previous study where 55 enucleations ( 11 primary and 44 secondary cases) were performed among 660 open globe injuries, only two patients $(0.3 \%)$ developed sympathetic ophthalmia. ${ }^{11}$ In our case, if the enucleation of the left eye was performed when ablepsia was unavoidable due to persistent high intraocular pressure after the second vitrectomy, sympathetic ophthalmia in the right eye may not have occurred. In the future, if a uveitis in the right eye recurs after the discontinuation of systemic steroid treatment, the enucleation of the left eye is an option to be considered.

\section{Conclusion}

Our case manifested sympathetic ophthalmia in the fellow eye that occurred after the vitrectomy performed to treat subretinal hemorrhage from PCV in the exciting eye. Bilateral occurrence of age-related macular degeneration and other inflammatory diseases should be carefully ruled out before making a diagnosis of sympathetic ophthalmia.

\section{Author contributions}

All authors contributed toward data analysis, drafting and critically revising the paper and agree to be accountable for all aspects of the work.

\section{Disclosure}

The authors report no conflicts of interest in this work.

\section{References}

1. Arevalo JF, Garcia RA, Al-Dhibi HA, Sanchez JG, Suarez-Tata L. Update on sympathetic ophthalmia. Middle East Afr JOphthalmol.2012;19(1):13-21. 
2. Guzman-Salas PJ, Serna-Ojeda JC, Guinto-Arcos EB, Pedroza-Seres M. Characteristics of sympathetic ophthalmia in a single international center. Open Ophthalmol J. 2016;10:154-159.

3. Aziz HA, Flynn HW, Young RC, Davis JL, Dubovy SR. Sympathetic ophthalmia: clinicopathologic correlation in a consecutive case series. Retina. 2015;35(8):1696-1703.

4. Tsaousis KT, Konidaris VE, Empeslidis T. Bilateral sensorineural hearing loss secondary to sympathetic ophthalmia in a human leukocyte antigen-A2 positive patient. Oman J Ophthalmol. 2017;10(3):232-234.

5. Chuang CT, Huang PS, Chen SC, Sheu SJ. Reversible alopecia in VogtKoyanagi-Harada disease and sympathetic ophthalmia. J Ophthalmic Inflamm Infect. 2013;3(1):41.

6. Wang Y, Chan CC. Gender differences in Vogt-Koyanagi-Harada disease and sympathetic ophthalmia. J Ophthalmol. 2014;2014:157803.
7. Davis JL, Mittal KK, Freidlin V, et al. HLA associations and ancestry in Vogt-Koyanagi-Harada disease and sympathetic ophthalmia. Ophthalmology. 1990;97(9):1137-1142.

8. Haruta M, Mukuno H, Nishijima K, Takagi H, Kita M. Sympathetic ophthalmia after 23-gauge transconjunctival sutureless vitrectomy. Clin Ophthalmol. 2010;4:1347-1349.

9. Kilmartin DJ, Dick AD, Forrester JV. Sympathetic ophthalmia risk following vitrectomy: should we counsel patients? Br J Ophthalmol. 2000;84(5):448-449.

10. Kilmartin DJ, Dick AD, Forrester JV. Prospective surveillance of sympathetic ophthalmia in the UK and Republic of Ireland. Br J Ophthalmol. 2000;84(3):259-263.

11. Savar A, Andreoli MT, Kloek CE, Andreoli CM. Enucleation for open globe injury. Am J Ophthalmol. 2009;147(4):595-600.

\section{Publish your work in this journal}

The International Medical Case Reports Journal is an international, peer-reviewed open-access journal publishing original case reports from all medical specialties. Previously unpublished medical posters are also accepted relating to any area of clinical or preclinical science. Submissions should not normally exceed 2,000 words or
4 published pages including figures, diagrams and references. The manuscript management system is completely online and includes a very quick and fair peer-review system, which is all easy to use. Visit http://www.dovepress.com/testimonials.php to read real quotes from published authors.

Submit your manuscript here: https://www.dovepress.com/international-medical-case-reports-journal-journal 